\title{
The Role of Estradiol in Idiopathic Anterior Uveitis during the Eumenorrheic Stage of Life
}

\author{
Ali A*, Thompson C, Wandel TL, Sharma S \\ Department of Ophthalmology, New York Medical College, USA \\ *Corresponding author: Amro Ali, MD, MO phth, Address: 40 Sunshine Cottage Road, Valhalla, NY 10595, New York, USA
}

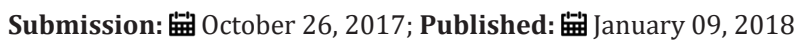

\begin{abstract}
Context: Women with idiopathic anterior uveitis do not appear to relapse while pregnant. Relapses are reported more frequently and intensely during premenstrual and menstrual stages. These observations suggest that uveitis may be related to fluctuations of sex steroid hormones.

Aims: To assess the effect of female sex steroid hormones on the course of idiopathic anterior uveitis.

Settings and Design: Comparative study done by retrospective chart review assessing the course of anterior uveitis for 60 patients (30 women each from eumenorrheic and postmenopausal stages) recently seen in uveitis clinic.

Methods and material: Retrospective review over one year from the date of the most recent visit after Institutional Review Board approval was obtained. Main outcome measurements were based on severity and duration of the intraocular inflammation. Other outcome measurements included recurrence of flare-up and response to treatment regimens, including steroids and immunomodulatory agents.
\end{abstract}

Statistical analysis: Differences between the number of patients in groups were tested for significance using a chi-square analysis.

Results: A higher incidence of uveitis flare-up occurred during the eumenorrheic stage compared to postmenopausal stage of life. The course of uveitis was of markedly increased severity in the eumenorrheic stage.

Conclusion: The incidence and intensity of uveitis flare-up is more pronounced during the active reproductive period of life. Differences in typical treatment may reflect the severity of the flare-ups or relate to concerns for side effect profiles amongst the groups. Differences in sexual hormone milieu are likely a major factor contributing to the differences in presentation of uveitis amongst eumenorrheic and postmenopausal women.

Keywords: Women; Estradiol; Menopause; Idiopathic; Uveitis

\section{Key Messages}

Uveitis occurs more frequently and more intensely during the eumenorrheic stage due to fluctuations in sex steroid hormones, such as estradiol. Blocking fluctuations in sex steroid hormones, therefore, has the potential to treat idiopathic anterior uveitis. Further studies are needed to assess the value of blocking fluctuations in sex steroid hormones on the course of idiopathic anterior uveitis.

\section{Introduction}

Non-infectious uveitis is the most common form of uveitis observed in the United States, and approximately $81 \%$ of noninfectious uveitis cases were diagnosed as anterior non-infectious uveitis. Anterior non-infectious uveitis is an autoimmune phenomenon, classified further into idiopathic autoimmune uveitis or uveitis secondary to systemic autoimmune disease. Idiopathic autoimmune uveitis is the single most common cause of uveitis. [1]

Autoimmune disorders affect nearly 20 million Americans. [2] Many of these disorders are found to be more common in women than men. Brandt el al and Clayton el al reported that 60 to $80 \%$ of those affected by autoimmune disorders are women. These autoimmune disorders include rheumatoid arthritis, multiple sclerosis, systemic lupus erythematosus, Graves' disease, and Hashimoto's disease. [3, 4] Similar to other autoimmune diseases, women have a higher prevalence of anterior noninfectious uveitis than men. [1] There is a significant body of literature on the biological differences between men and women that may affect the course of autoimmune disease. Differences in female sex steroid hormones are likely responsible for the dissimilarity in disease course between males and females.

Previous studies have showed that fluctuation of estrogen and progesterone affects inflammatory status, as seen with higher serum levels of human serum-CRP and the pro-inflammatory cytokines interleukin-6, interleukin-1, and tumor necrosis factoralpha. [5-8] Acharya et al demonstrated that women have been reported to demonstrate more uveitis relapses during the premenstrual and menstrual phases of their cycles. [9] In addition, on our Uveitis Service we have observed that many pregnant women did not relapse during the course of their pregnancy, and instead experienced a severe relapse two to four months after giving 
birth. (unpublished data) We hypothesize that the changes in the prevalence of autoimmune disorders as well as in the severity of uveitis course along different reproductive stages in women could possibility be attributed to female sex steroid hormones fluctuations.

In this study, we explicated the role of female sex steroid hormones on the course of uveitis through comparing the course of idiopathic anterior uveitis between eumenorrheic and postmenopausal stages. The outcome of this study cannot be generalized to the whole population as our sample included only females, which affects the external validity of the study. However, the study may assist in the determination of new methods to manage anterior uveitis, such as the use of topical estradiol for idiopathic autoimmune anterior uveitis.

\section{Materials and Methods}

After the Institutional Review Board approved this study and waived consent based on the retrospective nature of the study, the study was initiated. The study is composed of two groups (Groups A \& B) and each group contained 30 patients. Group A consisted of 30 women in the eumenarche stage (between 18 and 50 years old or menstruation for one year, whichever came first) and Group B which consisted of 30 women in the postmenopausal stage (more than 50 years old or one year after the cessation of menstruation, whichever came first).

The patients to be included in this study were determined by systematic randomization of uveitis patients with an established diagnosis of noninfectious idiopathic anterior uveitis. Infectious causes were excluded based on the appropriate tools including history, examination, and a battery of investigation ruling out the most common causes of infectious anterior uveitis based on clinical suspicion such as HIV, CMV, syphilis, tuberculosis, toxoplasmosis, and cat scratch disease. The data for the 60 included patients was reviewed retrospectively for 12 months from the date of their most recent visit. The factors reviewed included: the course of disease, the number and severity of relapses, the duration of each relapse, and the treatments administered to control inflammation.

Diagnosis of anterior uveitis is based on clinical presentation such as pain, redness, photophobia and presence of evidences of intraocular inflammation such as presence of inflammatory cells in anterior chamber and presence of synechiae for chronic cases.

The evaluation of the flare-up severity was determined based on two main factors:

1. The presence of inflammatory cells in the anterior chamber and the grade according to SUN criteria, and

2. The average flare-up duration in days.

The treatment for uveitis was also recorded, which included topical steroid eye drops, oral systemic steroids, and immunomodulatory therapy (IMT) (including methotrexate, mycophenolic acid, infliximab, and adalimumab). (Figures 1-3) Any intervention that may have affected the physiology of the sex hormones during the reproductive cycle such as the use any form of contraceptive pill or device would have excluded the patient from the study. The use of oral systemic steroids and immunomodulatory therapy (IMT) were also considered confounding factors, which may have affected the outcome of the study. This could have been avoided using stratification of the samples and matching the number of patients using systemic steroids and immunomodulatory therapy (IMT) in both groups of the study. This could have balanced the confounding factors, but was not performed as it could have also affected the internal validity of the study.

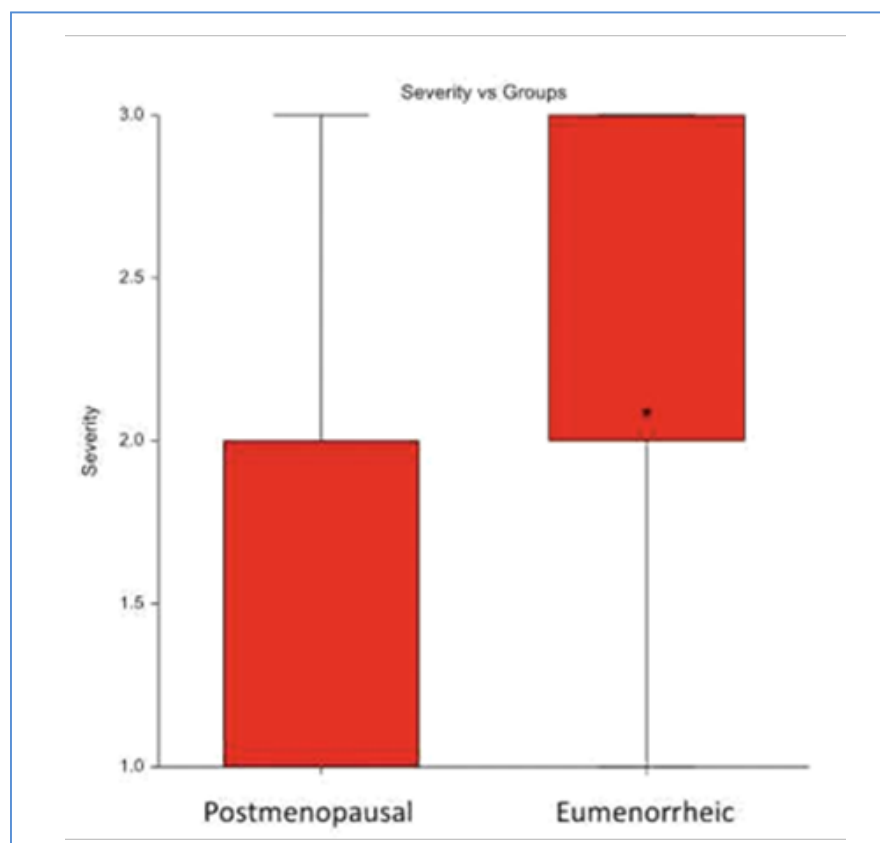

Figure 1: Impact of Female Sex Steroid Hormones on Uveitis flare Up Severity.

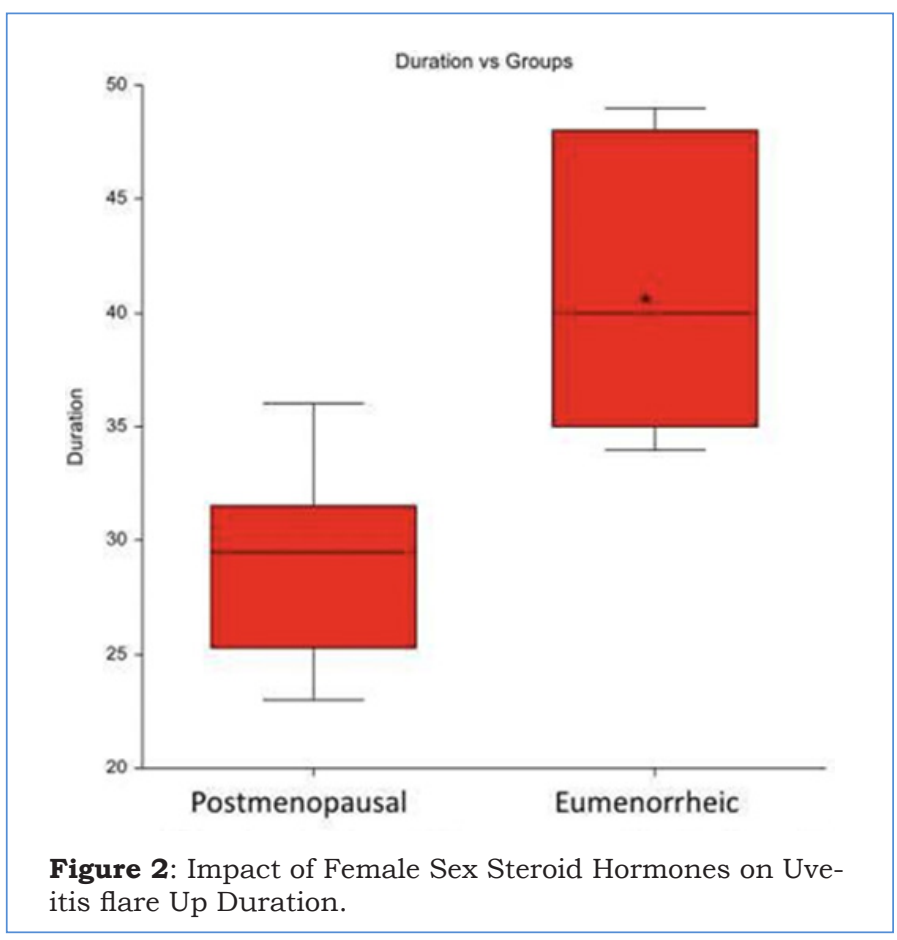




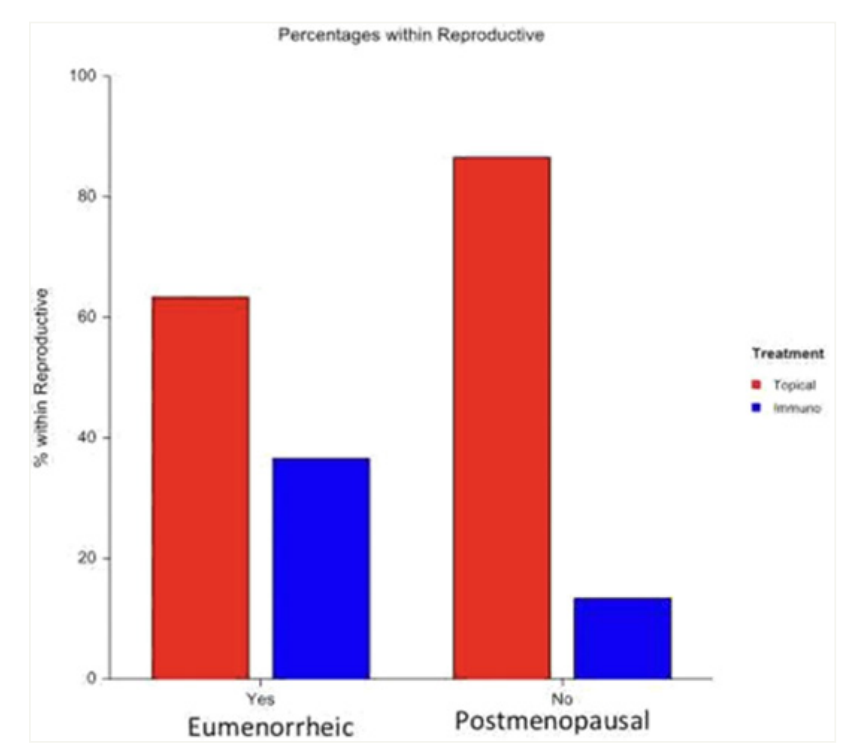

Figure 3: Impact of Female Sex Steroid Hormones on Topical Steroid vs. IMT.

In group A, all 30 eumenorrheicstage women who were recruited were asked routinely at every visit about their menstrual period and the use of any contraceptive measure, but no pregnancy tests

\section{Results}

were conducted for verification. In group B, all 30 postmenopausal stage women who were recruited were asked routinely at every visit about the use of any hormonal replacement therapy.

All efficacy variables were evaluated at baseline and during follow-up visits by slit-lamp biomicroscopy. The primary efficacy variables were anterior chamber cells graded according to SUN Criteria. Safety variables monitored included: age, reproductive stage of life which is defined based on age and last menstrual period, different lines of treatment such as topical steroid, systemic steroid, immunomodulatory agents, and other ophthalmologic findings. Others findings such as intraocular pressure, visual acuity, cystoid macular edema, posterior capsule opacification, etc., we're not assessed for in this study, as the main focus here was the relationship between intraocular inflammation and female sex hormones. All patients were evaluated by ophthalmologists on our uveitis team.

Different numbers between the groups were tested for significance using a chi-square analysis with $\mathrm{P}<0.05$ being a significant difference. Differences of variables between groups were determined utilizing a group t-test with $\mathrm{P}<0.05$ considered significant where appropriate. All data are presented as means +/standard error of means (SEM).

Table 1: Impact of female sex steroids on the incidence of uveitis flare-up.

\begin{tabular}{|c|c|c|c|c|}
\hline Reproductive Status & Flare-Ups & No Flare-Ups & Total & $\begin{array}{c}\text { Proportion (Flare- } \\
\text { Ups/Total) }\end{array}$ \\
\hline Postmenopausal & 7 & 23 & 30 & P1=.23 \\
\hline Eumenorrheic & 16 & 14 & 30 & $\mathrm{P} 2=.53$ \\
\hline
\end{tabular}

Group t-test shows a statistically significant difference in the incidence of uveitis flare-up between eumenorrheic and postmenopausal groups. The unique sexual hormone milieu of each group likely contributed to this difference. P1/P2 represents each group's proportions, postmenopausal to eumenorrheic, calculated. Two-Sided Tests of the Difference (P1 - P2) were calculated where H0: P1 = P2 vs. Ha: P1 $\neq \mathrm{P} 2$. Ho was rejected at $\mathrm{a} \leq 0.05$.

Table 2: Proportions Analysis on the incidence of uveitis flare-up between eumenorrheic vs postmenopausal women.

\begin{tabular}{|c|c|}
\hline Group 1 Event Rate (P1) & 0.23 \\
\hline Group 2 Event Rate (P2) & 0.53 \\
\hline Absolute Risk Difference [P1-P2] & 0.3 \\
\hline Number Needed to Treat 1/[P1-P2] & 3.33 \\
\hline Relative Risk Reduction [P1-P2]/P2 & 0.56 \\
\hline Relative Risk P1/P2 & 0.44 \\
\hline Odds Ratio 01/02 & 0.27 \\
\hline
\end{tabular}

Eumenorrheic vs postmenopausal women have nearly twice as high a proportion $(.53$ vs $.23, \mathrm{a}<.05)$ of uveitis flare-up, with a relative risk of .44 .

In our study, eumenorrheic women have nearly twice as high a proportion of uveitis flare-ups than postmenopausal women (.53 vs $.23, \alpha<.05$ ), with a relative risk of .44. (Table 1) Group t-test shows a statistically significant difference in the incidence of uveitis flareups between eumenorrheic and postmenopausal groups. (Table 2) The severity of flare-ups was measured using the severity grade of inflammation as defined by SUN criteria. [10] On cell grade scale, the median for the eumenorrheic group was 2 vs. 1 in the postmenopausal group $(\alpha<.05)$. (Table 3) (Figure 1) Group t-test shows a statistically significant difference in the mean cell grade between eumenorrheic and postmenopausal groups. (Table 4)

Table 3: Impact of female sex steroids on the severity of uveitis flare-up- median as standard of measure.

\begin{tabular}{|l|l|l|l|l|}
\hline Reproductive Status & Count & Median & $\mathbf{9 5 . 0 \%}$ LCL & $\mathbf{9 5 . 0 \%}$ UCL \\
\hline Eumenorrheic & 16 & 2 & 1 & 3 \\
\hline Postmenopausal & 7 & 1 & 1 & 2 \\
\hline
\end{tabular}

Cell grade, as defined by SUN criteria, is used here to describe severity of the flare-up. (20) Median cell grade is calculated for both eumenorrheic and postmenopausal groups. Group t-test shows a statistically significant difference in the median cell grade between eumenorrheic and postmenopausal groups. Ho was rejected at $a \leq 0.05$. Eumenorrheic-Postmenopausal. This result describes the average severity of uveitis flare-up as more intense in eumenorrheic compared to postmenopausal women, likely due to differences in sexual hormones.

Table 4: Variance analysis on severity of uveitis flare-up- median as standard of measure.

\begin{tabular}{|c|c|c|c|}
\hline $\begin{array}{c}\text { Variance } \\
\text { Assumption }\end{array}$ & $\begin{array}{c}\text { Mean } \\
\text { Difference }\end{array}$ & Standard Error & $\begin{array}{c}\mathbf{9 5 . 0 \%} \text { LCL- } \\
\text { UCL of } \boldsymbol{\mu} \mathbf{1} \boldsymbol{\mu} \mathbf{2}\end{array}$ \\
\hline Equal & -0.64 & 0.29 & -1.22 \\
\hline Unequal & -0.64 & 0.3 & -1.28 \\
\hline
\end{tabular}


Table 5: Variance analysis on severity of uveitis flare-up- median as standard of measure.

\begin{tabular}{|c|c|c|c|c|}
\hline $\begin{array}{c}\text { Reproductive } \\
\text { Status }\end{array}$ & Count & Median & $\mathbf{9 5 . 0 \% ~ L C L ~}$ & $\begin{array}{c}\mathbf{9 5 . 0 \%} \\
\text { UCL }\end{array}$ \\
\hline Eumenorrheic & 16 & 40 & 34 & 49 \\
\hline Postmenopausal & 7 & 29.5 & 25 & 30 \\
\hline
\end{tabular}

The mean duration, in days, is calculated for the both eumenorrheic and postmenopausal group.

The median duration of uveitis flare-up in days is calculated for the both eumenorrheic and postmenopausal groups. Uveitis flare-up in the eumenorrheic group averages a more prolonged course $(40.5 \pm 6)$ when compared to the postmenopausal group (29 \pm 4 ). (Table 5) (Figure 2) Group t-test shows a statistically significant difference in the average duration of flare-up between eumenorrheic and postmenopausal women (Table 6).

Table 6: Variance analysis on the duration, in days, of uveitis flare-up- median as standard of measure.

\begin{tabular}{|c|c|c|c|}
\hline $\begin{array}{c}\text { Variance } \\
\text { Assumption }\end{array}$ & $\begin{array}{c}\text { Mean } \\
\text { Difference }\end{array}$ & Standard Error & $\begin{array}{c}\mathbf{9 5 . 0 \%} \text { LCL-UCL } \\
\text { of } \boldsymbol{\mu 1} \mathbf{\mu} \mathbf{2}\end{array}$ \\
\hline Equal & -11.57 & 2.12 & -8.86 \\
\hline Unequal & -11.57 & 2.57 & -11.83 \\
\hline
\end{tabular}

Group t-test shows a statistically significant difference in the median duration of flare-up between eumenorrheic and postmenopausal women. Ho was rejected at $\alpha \leq 0.05$. $\mu 1-\mu 2$ : Eumenorrheic-Postmenopausal.Uveitis flare-up in the eumenorrheic group average a more prolonged course when compared to the postmenopausal group. This increase in duration is likely impacted in part by differences in sexual hormones.

When reviewing choice of treatment, we found that postmenopausal women typically received topical steroids $(26 / 30$ vs. 19/30), with less systemic steroid (1/30 vs. 16/30) and IMT (4/30 vs. $11 / 30)$ as compared to eumenorrheic women (Table 7). In addition, treatment of postmenopausal women is primarily steroid preparation without IMT (26/30 vs. 26/30) rather than with IMT (4/30 vs. 11/30) relative to eumenorrheic women (Table 8). Group t- test shows a statistically significant difference in the choice of topical steroids over IMT as treatment for uveitis flare-up in eumenorrheic and postmenopausal groups (Table 9). The proportion of women that received topical steroids over IMT was significantly greater for postmenopausal than eumenorrheic women $(.87$ vs $.63, \alpha<.05)$ with a relative risk of .73. (Tables $10 \&$ 11) (Figure 3) A short course of oral systemic steroids was used more frequently in the reproductive stage of life at 53\% (16/30) in comparison to $3 \%(1 / 30)$ in the postmenopausal stage (Table 7). Eumenorrheic women received a significantly greater proportion of systemic to topical steroids. (Tables 12-14) (Figure 4) Group t-test shows no statistically significant difference between the use of systemic steroid vs. IMT in uveitis flare during eumenorrheic stage compared to postmenopausal stage. (Tables 15-17) (Figure $5)$.

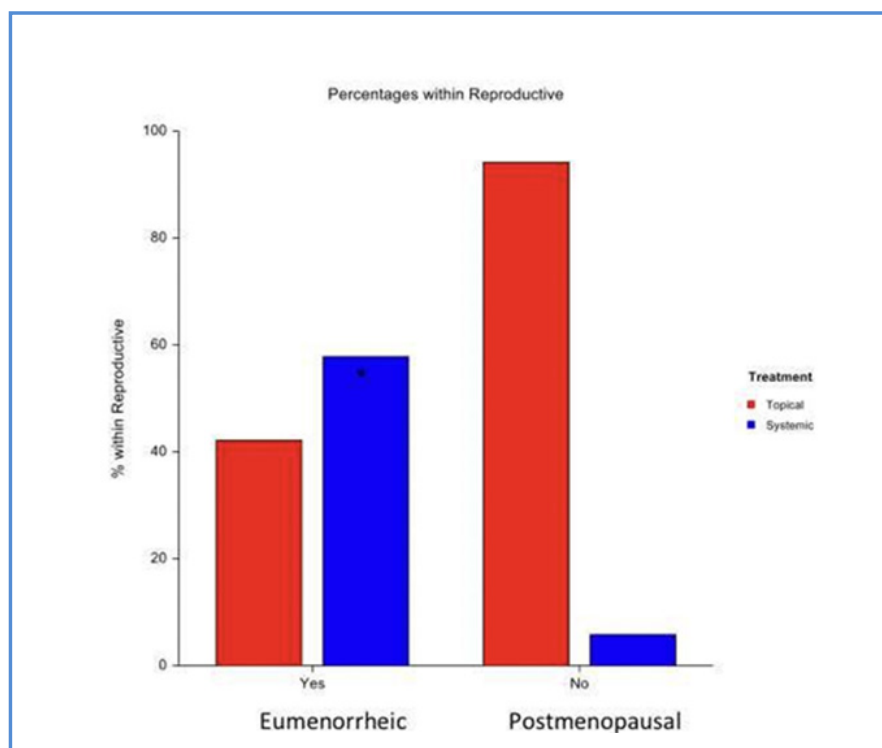

Figure 4: Impact of Female Sex Steroid Hormones on Topical Steroid vs. Systemic Steroid.

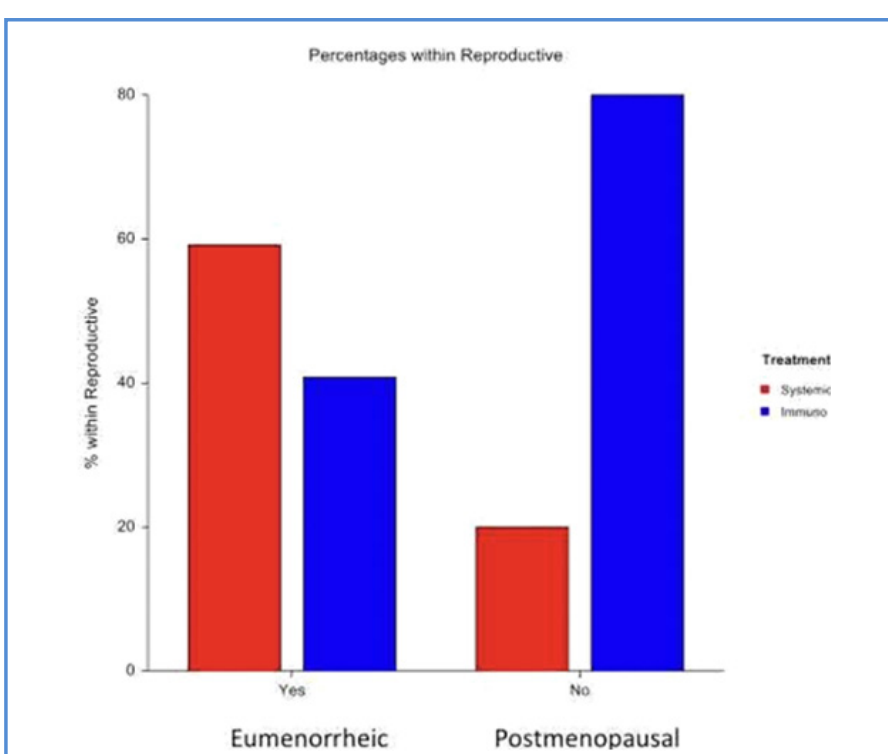

Figure 5: Impact of Female Sex Steroid Hormones on IMT Vs. Systemic Steroid.

\section{Discussion}

The study demonstrated a statistically significant difference in the incidence of uveitis flare-ups between eumenorrheic and postmenopausal groups. (Tables 1-2) The difference in uveitis course may relate to the unique sexual hormone milieu of each group. [9] This may correspond to monthly female sex hormone fluctuations during the menstrual cycle in eumenorrheic patients. We also demonstrated an average severity of uveitis flare-up that was more intense in eumenorrheic compared to postmenopausal women. (Tables 3-4) (Figure 1) Further, the average duration of uveitis flare-up was longer in eumenorrheic vs. postmenopausal women. (Tables 5-6) (Figure 2).

There is an association between an increase in the severity and duration of uveitis flare-up and fluctuations of female sex steroid 
hormones. Severe and prolonged uveitis flare-up would require more aggressive treatment than topical steroids alone, such as systemic steroid and IMT. Physicians prescribed both groups topical steroids more frequently than IMT and systemic steroids. (Tables 7-9) However, a significantly greater proportion of the eumenorrheic group received IMT or systemic steroids over topical steroids when compared to the postmenopausal group. (Tables 10-14) (Figures 3-4) The choice to use more aggressive treatment modalities in the eumenorrheic group suggests more severe and prolonged flare-ups in this group. The treatment choice may also reflect medication side effect profiles specific to each age group.

Table 7: Impact of female sex steroid hormones on treatment of uveitis flare ups.

\begin{tabular}{|c|c|c|}
\hline & Eumenorrheic & Postmenopausal \\
\hline Topical Steroid & $19 / 30$ & $26 / 30$ \\
\hline Systemic Steroid & $16 / 30$ & Jan-30 \\
\hline IMT & Nov-30 & Apr-30 \\
\hline
\end{tabular}

Table 8: Steroid Treatment without IMT Vs. IMT in treatment of Uveitis.

\begin{tabular}{|c|c|c|}
\hline & Eumenorrheic & Postmenopausal \\
\hline $\begin{array}{c}\text { Steroid Treatment } \\
\text { without IMT }\end{array}$ & $19 / 30$ & $26 / 30$ \\
\hline IMT & Nov-30 & Apr-30 \\
\hline
\end{tabular}

Table 9: Topical Steroids vs IMT in Treatment of Uveitis.

\begin{tabular}{|c|c|c|c|c|}
\hline $\begin{array}{c}\text { Reproductive } \\
\text { Status }\end{array}$ & Topical & IMT & Total & Proportion \\
\hline Eumenorrheic & 19 & 11 & 30 & P1 $=.63$ \\
\hline Postmenopausal & 26 & 4 & 30 & P2 $=.87$ \\
\hline
\end{tabular}

Table 10: Proportions Analysis on the use of topical steroid vs. IMT.

\begin{tabular}{|c|c|}
\hline Group 1 Event Rate (P1) & 0.63 \\
\hline Group 2 Event Rate (P2) & 0.87 \\
\hline Absolute Risk Difference [P1-P2] & 0.23 \\
\hline Number Needed to Treat 1/[P1-P2] & 4.29 \\
\hline Relative Risk Reduction [P1-P2]/P2 & 0.27 \\
\hline Relative Risk P1/P2 & 0.73 \\
\hline Odds Ratio 01/02 & 0.27 \\
\hline
\end{tabular}

Table 11: Median of topical steroid vs IMT use and confidence level [P1-P2].

\begin{tabular}{|c|c|c|c|c|}
\hline $\begin{array}{c}\text { Confidence } \\
\text { Interval }\end{array}$ & $\mathbf{P 1}$ & $\mathbf{P 2}$ & $\begin{array}{c}\text { Difference } \\
\text { P1-P2 }\end{array}$ & $\mathbf{9 5 . 0 \%}$ LCL-UCL of \\
\hline Name & & & & P1-P2 \\
\hline Wald Z & 0.63 & 0.87 & -0.23 & -4.71 \\
\hline
\end{tabular}

Ho was rejected at $\mathrm{a} \leq 0.05$. P1-P2.

Although in absolute numbers eumenorrheic women received more systemic steroids and postmenopausal women received more IMT (Table 7), the study did not have sufficient power to show a statistically significant difference $(\alpha \geq .05)$ between systemic steroids vs. IMT use in uveitis flare during eumenorrheic stage compared to postmenopausal stage. (Tables 15-17) (Figure 5). Low $\alpha(\alpha \geq 05)$ requires a greater sample size to increase the power of the study. As we have seen the incidence of flare-ups is lower in the postmenopausal group, studying a larger group of postmenopausal women may demonstrate a statistically significant difference between IMT and/or steroid treatment, but would require a larger pool of patients.

Table 12: Topical Steroids vs Systemic Steroids in Treatment of Uveitis.

\begin{tabular}{|c|c|c|c|c|}
\hline Reproductive Status & Topical & Systemic & Total & Proportion(Topical/Total) \\
\hline Eumenorrheic & 19 & 26 & 45 & $\mathrm{P} 1=.42$ \\
\hline Postmenopausal & 16 & 1 & 17 & $\mathrm{P} 2=.94$ \\
\hline
\end{tabular}

Group t-test shows a statistically significant difference in the choice of steroid modality used to treat eumenorrheic and postmenopausal groups*. Physicians used topical steroids more frequently than systemic steroids in each group. Overall, eumenorrheic women received more topical steroids than postmenopausal women. Further, eumenorrheic women received more systemic steroids than postmenopausal women. Treatment choice may have been influenced by increased: incidence, average severity, and average duration, of flare ups in the eumenorrheic group that prompted more aggressive modalities.

Table 13: Proportions Analysis on the use of topical steroid vs. Systemic Steroid.

\begin{tabular}{|c|c|}
\hline Group 1 Event Rate (P1) & 0.42 \\
\hline Group 2 Event Rate (P2) & 0.94 \\
\hline Absolute Risk Difference [P1-P2] & 0.52 \\
\hline Number Needed to Treat 1/[P1-P2] & 1.93 \\
\hline Relative Risk Reduction [P1-P2]/P2 & 0.55 \\
\hline Relative Risk P1/P2 & 0.45 \\
\hline Odds Ratio 01/02 & 0.05 \\
\hline
\end{tabular}

Table 14: Confidence Intervals of topical vs systemic steroid use and confidence level [P1-P2].

\begin{tabular}{|c|c|c|c|c|}
\hline $\begin{array}{c}\text { Confidence } \\
\text { Interval }\end{array}$ & P1 & P2 & $\begin{array}{c}\text { Difference } \\
\text { P1-P2 }\end{array}$ & $\mathbf{9 5 . 0 \%}$ LCL-UCL of \\
\hline Name & & & & P1-P2 \\
\hline Wald Z & 0.42 & 0.94 & -0.52 & -0.44 \\
\hline
\end{tabular}

Ho was rejected at $\mathrm{a} \leq$ 0.05. P1-P2.

The decrease in flare-up incidence, severity, and duration in postmenopausal women suggests that uveitis in postmenopausal women is of low severity level. (Tables $1,3,5$ ) This may be related to 
a more quiescent immune system with aging or the lack of hormonal changes that are prominent during the reproductive period of life.

Table 15: The use of IMT and steroid vs steroid alone during eumenorrheic and postmenopausal stage.

\begin{tabular}{|c|c|c|c|c|}
\hline $\begin{array}{c}\text { Reproductive } \\
\text { Status }\end{array}$ & Systemic & IMT & Total & $\begin{array}{c}\text { Proportion(Systemic/ } \\
\text { Total) }\end{array}$ \\
\hline Eumenorrheic & 16 & 11 & 27 & P1 $=.59$ \\
\hline Postmenopausal & 1 & 4 & 5 & P2 $=.20$ \\
\hline
\end{tabular}

Table 16: Proportions Analysis on the use of IMT vs. Systemic Steroid.

\begin{tabular}{|c|c|}
\hline Group 1 Event Rate (P1) & 0.59 \\
\hline Group 2 Event Rate (P2) & 0.2 \\
\hline Absolute Risk Difference [P1-P2] & 0.39 \\
\hline Number Needed to Treat 1/[P1-P2] & 2.55 \\
\hline Relative Risk Reduction [P1-P2]/P2 & 1.96 \\
\hline Relative Risk P1/P2 & 2.96 \\
\hline Odds Ratio 01/02 & 5.82 \\
\hline
\end{tabular}

Table 17: Confidence Intervals of IMT vs systemic steroid use and confidence level (P1-P2).

\begin{tabular}{|c|c|c|c|c|}
\hline $\begin{array}{c}\text { Confidence Interval } \\
\text { Name }\end{array}$ & P1 & P2 & $\begin{array}{c}\text { Difference } \\
\text { P1-P2 }\end{array}$ & $\begin{array}{c}\text { 95.0\% LCL- } \\
\text { UCL of }\end{array}$ \\
\hline Wald Z & 0.59 & 0.2 & 0.39 & P1-P2 \\
\hline
\end{tabular}

Ho was not rejected at $\mathrm{a} \leq 0.05$. P1-P2.

Our study shows several significant differences amongst eumenorrheic and postmenopausal women. The unique hormonal milieu of eumenorrheic women, especially estradiol and proinflammatory cytokines, likely contributed to the increases in flare-up incidence, severity, and duration seen in the eumenorrheic group. (Figures 1-2) (Tables 1,3,5) Further prospective studies are needed to correlate the role of female sex hormones on the course of uveitis, mainly estradiol and its potential to treat autoimmune uveitis.

Our study showed the incidence of idiopathic anterior nonnfectious uveitis is higher during the active reproductive stage of life. This higher incidence of flare-ups is likely what guides wider usage of short course systemic steroids in eumenorrheic patient groups; to ameliorate and reduce further flare-ups. Further, the course of uveitis in eumenorrheic women is more severe with longer duration. By comparison, uveitis in postmenopausal women is less severe with shorter duration. Uveitis flares in postmenopausal women typically involve lower-grade inflammation intensity, with greater response to topical steroids. Sex hormones, including estradiol and progesterone, and the oxidative properties associated with them seem to be major players in the pathophysiology of uveitis. The higher level of estradiol seen in eumenorrheic women as compared to postmenopausal women may contribute to greater incidence and severity of uveitis.

\section{References}

1. Thorne JE, Suhler E, Skup M, Tari S, Macaulay D, et al (2016) Prevalence of Noninfectious Uveitis in the United StatesA Claims-Based Analysis. JAMA Ophthalmol 134(11): 1237-1245.

2. Cooper GS, Bynum MLK, Somers EC (2009) Recent Insights in the Epidemiology of Autoimmune Diseases: Improved Prevalence Estimates and Understanding of Clustering of Diseases. Journal of autoimmunity 33(3-4): 197-207.

3. Ji J, Sundquist J, Sundquist K (2016) Gender specific incidence of autoimmune diseases from national registers. Journal of Autoimmunity 69: 102-106.

4. Brandt JE, Priori R, Valesini G, Fairweather DL (2015) Sex differences in Sjogren's syndrome: a comprehensive review of immune mechanisms. Biology of Sex Differences 6:19.

5. Pude JJ, Blum CA (2006) Menstrual cycle symptoms are associated with changes in low-grade inflammation. European Journal of Clinical Investigation 36(1): 58-64.

6. Kop WJ, Gottdiener JS (2005) The role of immune system parameters in the relationship between depression and coronary artery disease. Psychosom Med 67(Suppl 1): S37-S41.

7. Ford DE, Erlinger TP (2004) Depression and C-reactive protein in US adults: data from the Third National Health and Nutrition Examination Survey. Arch Intern Med 164 (9): 1010-1014.

8. Tuglu C, Kara SH, Caliyurt O, Vardar E, Abay E (2003) Increased serum tumor necrosis factor-alpha levels and treatment response in major depressive disorder. Psychopharmacology (Berl) 170(4): 429-433.

9. Sanghvi C, Aziz K, Jones NP (2004) Uveitis and the menstrual cycle. Eye 18(5): 451-454.

10. Jabs DA, Nussenblatt RB, Rosenbaum JT (2005) Standardization of Uveitis Nomenclature (SUN) Working Group. Standardization of Uveitis Nomenclature for Reporting Clinical Data. Results of the First International Workshop. Am J Ophthalmol 140(3): 509-516. 